\title{
A Parametric Approach to Solve Multi Objective Fuzzy Linear Programming Problem
}

\author{
Suraj Singh Chand \\ Dept. of Mathematics \\ Graphic Era Hill University \\ Dehradun, Uttarakhand, India
}

\author{
Vineet Bhatt \\ Dept. of Mathematics \\ Graphic Era Hill University \\ Dehradun, Uttarakhand, India
}

\begin{abstract}
A new method has been proposed by Senthilkumar and Rajendran [1] for solving fuzzy linear programming problem in parametric form. This paper extends this method for solving multi-objective fuzzy linear programming problem with fuzzy variables in parametric form. To obtain optimal solution, problem is converted into two auxiliary crisp linear programming problems corresponding to each objective functions. A numerical example is given to check the feasibility of the proposed method.
\end{abstract}

Keywords: Multi objective fuzzy linear programming, fuzzy number.

\section{INTRODUCTION}

The concept of decision making in fuzzy environment is proposed by Bellman and Zadeh [2]. Zimmermann [3] proposed the first formulation of fuzzy linear programming. Subsequently, a number of researchers have studied various types of fuzzy linear programming problems, such as many authors solved these problems [4], [5], [6] by using different approaches. Allahviranloo et.al.[7] proposed a new method for solving fully fuzzy linear programming problem by applying the concept of comparison of fuzzy numbers. Kumar and Kaur [8] introduced a new method for solving fuzzy linear programming problem with trapezoidal fuzzy numbers. Roseline and Amirtharaj [9] solved a fuzzy linear programming problem by using $\alpha$-cut and ranking function. Cadenas and Verdegay [10] studied a linear programming problem in which all its elements are defined as fuzzy sets. Senthilkumar and Rajendran [1] solved fuzzy linear programming problem in parametric form. Stanciulescu et.al[11] proposed a new method in which fuzzy decision variables have been considered for solving multi objective fuzzy linear programming problems. Cadenas and Verdegay [12] solved multi objective fuzzy linear programming problem by using ranking function. In this work, a new method has been proposed to solve multi-objective linear programming problems with fuzzy variables in parametric form. The constant on right hand side have also been considered as fuzzy numbers in parametric form. One numerical example has been presented to illustrate the proposed method.

\section{PRELIMINARIES}

A review of some definitions related to the present work is as follows:

Definition: A fuzzy number is a map $f: R \rightarrow[0,1]$ which satisfies [13]:

1. $\mathrm{f}$ is upper semi-continuous.

2. $\mathrm{f}(\mathrm{x})=0$, outside some interval $[\mathrm{c}, \mathrm{d}] \subset \mathrm{R}$.

3. There exist real numbers $a, b$ such that $\mathrm{c} \leq \mathrm{a} \leq \mathrm{b} \leq \mathrm{d}$, where

a) $\mathrm{f}(\mathrm{x})$ is monotonic increasing on $[\mathrm{c}, \mathrm{a}] \subset \mathrm{R}$.

b) $\mathrm{f}(\mathrm{x})$ is monotonic decreasing on $[\mathrm{b}, \mathrm{d}] \subset \mathrm{R}$.

c) $\mathrm{f}(\mathrm{x})=1 \forall \mathrm{x} \in[\mathrm{a}, \mathrm{b}]$.

Definition: An arbitrary fuzzy number in parametric form [14] is represented by an ordered pair of functions $(\underline{\mathrm{f}}(\mathrm{r}), \overline{\mathrm{f}}(\mathrm{r})), 0 \leq \mathrm{r} \leq 1$, which satisfies following requirements:

1. $\underline{f}(r)$ is a bounded and monotonic increasing left continuous function over $[0,1]$.

2. $\overline{\mathrm{f}}(\mathrm{r})$ is a bounded and monotonic decreasing left continuous function over $[0,1]$.

3. $\underline{\mathrm{f}}(\mathrm{r}) \leq \overline{\mathrm{f}}(\mathrm{r}), 0 \leq \mathrm{r} \leq 1$.

Arithmetic on parametric fuzzy numbers:

Let $\mathrm{x}=(\underline{\mathrm{x}}(\mathrm{r}), \overline{\mathrm{x}}(\mathrm{r}))$ and $\mathrm{y}=(\mathrm{y}(\mathrm{r}), \overline{\mathrm{y}}(\mathrm{r}))$ be two fuzzy numbers in parametric form and $\mathrm{k}$ be a scalar then arithmetic on parametric fuzzy numbers defined as [14]:

1. $x=y$ iff $\underline{x}(r)=\underline{y}(r)$ and $\bar{x}(r)=\bar{y}(r)$.

2. $x+y=(\underline{x}(r)+\underline{y}(r), \bar{x}(r)+\bar{y}(r))$.

3. $\mathrm{kx}=(\mathrm{kx}(\mathrm{r}), \mathrm{k} \overline{\mathrm{x}}(\mathrm{r}))$ if $\mathrm{k}$ is non-negative and $\mathrm{kx}=(\mathrm{k} \overline{\mathrm{x}}(\mathrm{r}), \mathrm{k} \underline{\mathrm{x}}(\mathrm{r}))$ if $\mathrm{k}$ is negative.

Definition: A fuzzy linear programming problem in parametric form is defined as [1]:

$\operatorname{Max} / \operatorname{Min} \widetilde{z}=c \tilde{X}$

Subject to

$$
A \tilde{x} \leq(=\text { or } \geq) \tilde{b}, \tilde{x} \geq 0,
$$

where,

$c=\left(c_{1}, c_{2} \ldots c_{n}\right)$ is non-negative crisp vector,

$\mathrm{A}=\left[\mathrm{a}_{\mathrm{ij}}\right]_{\mathrm{m} \times \mathrm{n}}$ is non-negative crisp matrix,

$\tilde{\mathrm{x}}=\left(\tilde{\mathrm{x}}_{1}, \tilde{\mathrm{x}}_{2} \ldots \tilde{\mathrm{x}}_{\mathrm{n}}\right)$ and

$\tilde{\mathrm{b}}=\left(\tilde{\mathrm{b}}_{1}, \tilde{\mathrm{b}}_{2} \ldots \tilde{\mathrm{b}}_{\mathrm{m}}\right)$ are non-negative fuzzy vectors such that $\tilde{\mathrm{x}}_{\mathrm{j}}$ and $\tilde{b}_{i} 1 \leq \mathrm{j} \leq \mathrm{n}, 1 \leq \mathrm{i} \leq \mathrm{m}$ are fuzzy numbers in parametric form.

Definition: A multi objective fuzzy linear programming problem is defined as follows:

$\operatorname{Max} / \operatorname{Min} \mathrm{z}_{\mathrm{p}}(\tilde{\mathrm{x}}), \mathrm{p}=1,2 \ldots \mathrm{k}$

Subject to

$A \tilde{x} \leq(=$ or $\geq) \tilde{b}, \tilde{x} \geq 0$, 
where,

$\mathrm{z}_{\mathrm{p}}(\tilde{\mathrm{x}})=\mathrm{C}_{\mathrm{p}} \tilde{\mathrm{x}}$,

$C_{P}=\left(C_{P 1}, C_{P 2}, \ldots C_{P n}\right)$ are non-negative crisp vectors for $\mathrm{p}=1,2, \ldots \mathrm{k}$

$\mathrm{A}=\left[\mathrm{a}_{\mathrm{ij}}\right]_{\mathrm{m} \times \mathrm{n}}$ is non-negative crisp matrix,

$\tilde{\mathrm{x}}=\left(\tilde{\mathrm{x}}_{1}, \tilde{\mathrm{x}}_{2} \ldots \tilde{\mathrm{x}}_{\mathrm{n}}\right)$ and $\tilde{\mathrm{b}}=\left(\tilde{\mathrm{b}}_{1}, \tilde{\mathrm{b}}_{2} \ldots \tilde{\mathrm{b}}_{\mathrm{m}}\right)$ are non-negative fuzzy vectors such that $\tilde{\mathrm{x}}_{\mathrm{j}}$ and $\tilde{b}_{\mathrm{i}}, 1 \leq \mathrm{j} \leq \mathrm{n}, 1 \leq \mathrm{i} \leq \mathrm{m}$ are fuzzy numbers in parametric form.

\section{PROCEDURE TO FIND OPTIMAL SOLUTION FOR MULTI OBJECTIVE FUZZY LINEAR PROGRAMMING PROBLEMS [MOFLPP].}

Consider the multi objective fuzzy linear programming problem

$\operatorname{Max} / \operatorname{Min} z_{p}\left(\tilde{x}_{j}\right) p=1,2 \ldots k, j=1,2 \ldots n$

Subject to

$$
\begin{aligned}
& \mathrm{a}_{11} \tilde{\mathrm{x}}_{1}+\mathrm{a}_{12} \tilde{\mathrm{x}}_{2}+\ldots+\mathrm{a}_{1 \mathrm{n}} \tilde{\mathrm{x}}_{\mathrm{n}} \leq \tilde{\mathrm{b}}_{1} \\
& \mathrm{a}_{21} \tilde{\mathrm{x}}_{1}+\mathrm{a}_{22} \tilde{\mathrm{x}}_{2}+\ldots+\mathrm{a}_{2 \mathrm{n}} \tilde{\mathrm{x}}_{\mathrm{n}} \leq \tilde{\mathrm{b}}_{2} \\
& \vdots \quad \vdots \quad \vdots \\
& \mathrm{a}_{\mathrm{m} 1} \tilde{\mathrm{x}}_{1}+\mathrm{a}_{\mathrm{m} 2} \tilde{\mathrm{x}}_{2}+\ldots+\mathrm{a}_{\mathrm{mn}} \tilde{\mathrm{x}}_{\mathrm{n}} \leq \tilde{\mathrm{b}}_{\mathrm{m}} \\
& \tilde{\mathrm{x}}_{1}, \tilde{\mathrm{x}}_{2} \ldots \tilde{\mathrm{x}}_{\mathrm{n}} \geq 0 .
\end{aligned}
$$

The above MOFLPP can be written in standard form as

$\operatorname{Max} / \operatorname{Min} z_{p}\left(\underline{x}_{j}, \bar{x}_{j}\right)$ where $p=1,2 \ldots k$ and $j=1,2 \ldots n$

Subject to

$$
\begin{aligned}
& a_{i 1}\left(\underline{x}_{1}, \bar{x}_{1}\right)+\ldots+a_{i n}\left(\underline{x}_{n}, \bar{x}_{n}\right)=\left(\underline{b}_{i}(r), \bar{b}_{i}(r)\right), \\
& \text { for } i=1,2 \ldots m . \\
& \underline{x}_{j}, \bar{x}_{j} \geq 0, j=1,2 \ldots n .
\end{aligned}
$$

\subsection{Steps to Solve MOFLPP}

Step- I: Replace $r$ as 0 and 1 in (3.2) to get two auxiliary multi objective fuzzy linear programming problems.

Step- II: For $r=0$, (3.2) changes into crisp multi objective linear programming problem (MOLPP), as follows

$\operatorname{Max} / \operatorname{Min} z_{p}\left(\underline{x}_{j}^{0}, \bar{x}_{j}^{0}\right) \quad p=1,2, \ldots k$

Subject to

$a_{i 1}\left(\underline{x}_{1}^{0}, \bar{x}_{1}^{0}\right)+\ldots+a_{i n}\left(\underline{x}_{n}^{0}, \bar{x}_{n}^{0}\right) \leq\left(\underline{b}_{i}(0), \bar{b}_{i}(0)\right)$ for $\mathrm{i}=$ $1,2 \ldots \mathrm{m}$,

$\underline{x}_{j}^{0}, \bar{x}_{j}^{0} \geq 0$ for $j=1,2 \ldots n$.

Step-III: Split (3.3.) into two MOLPP corresponding to $\mathrm{r}=0$, which are as follow:

$\operatorname{Max} / \operatorname{Min} \mathrm{z}_{\mathrm{p}} \underline{x}_{\mathrm{j}}^{0} \quad \mathrm{i}=1,2 \ldots \mathrm{k}$

Subject to

$\mathrm{a}_{\mathrm{i} 1} \underline{\mathrm{x}}_{1}^{0}+\mathrm{a}_{\mathrm{i} 2} \underline{\mathrm{x}}_{2}^{0}+\ldots+\mathrm{a}_{\mathrm{in}} \underline{\mathrm{x}}_{\mathrm{n}}^{0} \leq \underline{\mathrm{b}}_{\mathrm{i}}(0)$

for $\mathrm{i}=1,2 \ldots \mathrm{m}$, $\underline{x}_{j}^{0} \geq 0$ for $j=1,2 \ldots n$,

and

$\operatorname{Max} / \operatorname{Min} \mathrm{z}_{\mathrm{p}} \overline{\mathrm{x}}_{\mathrm{j}}^{0} \quad \mathrm{p}=1,2 \ldots \mathrm{k}$

Subject to

$\mathrm{a}_{\mathrm{i} 1} \overline{\mathrm{x}}_{1}^{0}+\mathrm{a}_{\mathrm{i} 2} \overline{\mathrm{x}}_{2}^{0}+\ldots+\mathrm{a}_{\mathrm{in}} \overline{\mathrm{x}}_{\mathrm{n}}^{0} \leq \overline{\mathrm{b}}_{\mathrm{i}}(0)$

for $\mathrm{i}=1,2 \ldots \mathrm{m}$,

$\bar{x}_{j}^{0} \geq 0$ for $j=1,2 \ldots n$.

Solving (3.4) and (3.5) separately to obtain optimal solutions of two MOLPP for $\mathrm{r}=0$ as follows:

$\underline{x}_{1}^{0}, \underline{x}_{2}^{0}, \underline{x}_{3}^{0} \ldots \underline{x}_{n}^{0}$

$\overline{\mathrm{x}}_{1}^{0}, \overline{\mathrm{x}}_{2}^{0}, \overline{\mathrm{x}}_{3}^{0} \ldots \overline{\mathrm{x}}_{\mathrm{n}}^{0}$

Step-IV: Replace $r=1$ in (3.2) to get

$\operatorname{Max} / \operatorname{Min} \mathrm{z}_{\mathrm{p}}\left(\underline{x}_{\mathrm{j}}^{1}, \overline{\mathrm{x}}_{\mathrm{j}}^{1}\right) \quad \mathrm{p}=1,2 \ldots \mathrm{k}$

Subject to

$\mathrm{a}_{\mathrm{i} 1}\left(\underline{\mathrm{x}}_{1}^{1}, \overline{\mathrm{x}}_{1}^{1}\right)+\cdots+\mathrm{a}_{\mathrm{in}}\left(\underline{x}_{\mathrm{n}}^{1}, \overline{\mathrm{x}}_{\mathrm{n}}^{1}\right) \leq\left(\underline{\mathrm{b}}_{\mathrm{i}}(1), \overline{\mathrm{b}}_{\mathrm{i}}(1)\right)$

for $i=1,2 \ldots m, \underline{x}_{j}^{1}, \bar{x}_{j}^{1} \geq 0$ for $j=1,2 \ldots n$.

Step-V: Split (3.7) into two MOLPP corresponding to $r=1$ which are as follow:

$\operatorname{Max} / \operatorname{Min} \mathrm{z}_{\mathrm{p}} \underline{x}_{\mathrm{j}}^{1} \quad \mathrm{p}=1,2 \ldots \mathrm{k}$

Subject to

$\mathrm{a}_{\mathrm{i} 1} \underline{\mathrm{x}}_{1}^{1}+\mathrm{a}_{\mathrm{i} 2} \underline{\mathrm{x}}_{2}^{1}+\ldots+\mathrm{a}_{\mathrm{in}} \underline{\mathrm{x}}_{\mathrm{n}}^{1} \leq \underline{\mathrm{b}}_{\mathrm{i}}(1)$

for $i=1,2 \ldots m$ and $\underline{x}_{j}^{1} \geq 0$ for $j=1,2 \ldots n$,

and

$\operatorname{Max} / \operatorname{Min} z_{\mathrm{p}} \bar{x}_{j}^{1} \quad \mathrm{p}=1,2 \ldots \mathrm{k}$

Subject to

$\mathrm{a}_{\mathrm{i} 1} \overline{\mathrm{x}}_{1}^{1}+\mathrm{a}_{\mathrm{i} 2} \overline{\mathrm{x}}_{2}^{1}+\ldots+\mathrm{a}_{\mathrm{in}} \overline{\mathrm{x}}_{\mathrm{n}}^{1} \leq \overline{\mathrm{b}}_{\mathrm{i}}(1)$

for $i=1,2 \ldots m$ and $\bar{x}_{j}^{1} \geq 0$ for $j=1,2 \ldots n$

Solve (3.8) and (3.9) separately to obtain optimal solutions of two MOLPPs for $r=1$ as follows:

$$
\begin{aligned}
& \underline{x}_{1}^{1}, \underline{x}_{2}^{1}, \underline{x}_{3}^{1} \ldots \underline{x}_{n}^{1} \\
& \bar{x}_{1}^{1}, \bar{x}_{2}^{1}, \bar{x}_{3}^{1}, \ldots \bar{x}_{n}^{1}
\end{aligned}
$$

Step-VI: Now use optimal solutions (3.6) and (3.10) in

$$
\left.\begin{array}{l}
\overline{x_{j}}=\left(\overline{x_{j}^{1}}-\overline{x_{j}^{0}}\right) r+x_{j}^{0} \\
\overline{x_{j}}=\left(\overline{\overline{x_{j}^{1}}}-\overline{\overline{x_{j}^{0}}}\right) r+\overline{\overline{x_{j}^{0}}}
\end{array}\right\}
$$

Using these, calculate $\tilde{x}_{j}=\left(x_{j}, \overline{x_{j}}\right)$ for $j=1,2 \ldots n$

to obtain optimal solution of (3.2).

Step-VII: Finally substitute the values of $\tilde{x}_{j}, j=1,2 \ldots n$ in (3.2) to get optimal values for each objective function $z_{p}$, $\mathrm{p}=1,2 \ldots \mathrm{k}$.

\section{EXAMPLE}

Consider the following parametric form of MOFLPP

$\operatorname{Max} \mathrm{z}_{1}=\tilde{\mathrm{x}}_{1}+4 \tilde{\mathrm{x}}_{2}$ 
$\operatorname{Max} \mathrm{z}_{2}=2 \tilde{\mathrm{x}}_{1}+5 \tilde{\mathrm{x}}_{2}$

Subject to

$3 \tilde{\mathrm{x}}_{1}+\tilde{\mathrm{x}}_{2} \leq(30+\mathrm{r}, 42-\mathrm{r})$

$\tilde{\mathrm{x}}_{1}+7 \tilde{\mathrm{x}}_{2} \leq(22+\mathrm{r}, 32-\mathrm{r})$

$\tilde{\mathrm{x}}_{1}, \tilde{\mathrm{x}}_{2} \geq 0$ and $0 \leq \mathrm{r} \leq 1$.

The above MOFLPP can be written as:

$\operatorname{Max} \mathrm{z}_{1}=\left(\underline{\mathrm{x}_{1}}, \overline{\mathrm{x}_{1}}\right)+4\left(\underline{\mathrm{x}_{2}}, \overline{\mathrm{x}_{2}}\right)$

$\operatorname{Max} z_{2}=2\left(\underline{x_{1}}, \overline{x_{1}}\right)+5\left(\underline{x_{2}}, \overline{x_{2}}\right)$

Subject to

(4.1)

$3\left(\underline{x_{1}}, \overline{x_{1}}\right)+\left(\underline{x_{2}}, \overline{x_{2}}\right) \leq(30+r, 42-r)$

$\left(\underline{x_{1}}, \overline{x_{1}}\right)+7\left(\underline{x_{2}}, \overline{x_{2}}\right) \leq(22+r, 32-r)$.

$\underline{\mathrm{x}_{1}}, \overline{\mathrm{x}_{1}}, \underline{\mathrm{x}_{2}}, \overline{\mathrm{x}_{2}} \geq 0$ and $0 \leq \mathrm{r} \leq 1$

First replace $r$ as 0 then (4.1) becomes

$\operatorname{Max} \mathrm{z}_{1}=\left(\underline{\mathrm{x}}_{1}^{0}, \overline{\mathrm{x}}_{1}^{0}\right)+4\left(\underline{\mathrm{x}}_{2}^{0}, \overline{\mathrm{x}}_{2}^{0}\right)$

$\operatorname{Max} \mathrm{z}_{2}=2\left(\underline{x}_{1}^{0}, \overline{\mathrm{x}}_{1}^{0}\right)+5\left(\underline{\mathrm{x}}_{2}^{0}, \overline{\mathrm{x}}_{2}^{0}\right)$

Subject to

$3\left(\underline{\mathrm{x}}_{1}^{0}, \overline{\mathrm{x}}_{1}^{0}\right)+\left(\underline{\mathrm{x}}_{2}^{0}, \overline{\mathrm{x}}_{2}^{0}\right) \leq(30,42)$

$\left(\underline{x}_{1}^{0}, \bar{x}_{1}^{0}\right)+7\left(\underline{x}_{2}^{0}, \bar{x}_{2}^{0}\right) \leq(22,32)$

$\underline{x}_{1}^{0}, \bar{x}_{1}^{0}, \underline{x}_{2}^{0}, \bar{x}_{2}^{0} \geq 0$.

There are two auxiliary crisp Multi objective Linear programming Problem (MOLPP) corresponding to $r=0$

$\operatorname{Max} \mathrm{z}_{1}=\underline{\mathrm{x}}_{1}^{0}+4 \underline{\mathrm{x}}_{2}^{0}$

$\operatorname{Max} z_{2}=2 \underline{x}_{1}^{0}+5 \underline{x}_{2}^{0}$

Subject to

$3 \underline{x}_{1}^{0}+\underline{x}_{2}^{0} \leq 30$

$\underline{x}_{1}^{0}+7 \underline{x}_{2}^{0} \leq 22$

$\underline{\mathrm{x}}_{1}^{0}, \underline{\mathrm{x}}_{2}^{0} \geq 0$

and

$\operatorname{Max} \mathrm{z}_{1}=\overline{\mathrm{x}}_{1}^{0}+4 \overline{\mathrm{x}}_{2}^{0}$

$\operatorname{Max} \mathrm{z}_{2}=2 \overline{\mathrm{x}}_{1}^{0}+5 \overline{\mathrm{x}}_{2}^{0}$

Subject to

$3 \overline{\mathrm{x}}_{1}^{0}+\overline{\mathrm{x}}_{2}^{0} \leq 42$

$\overline{\mathrm{x}}_{1}^{0}+7 \overline{\mathrm{x}}_{2}^{0} \leq 32$

$\overline{\mathrm{x}}_{1}^{0}, \overline{\mathrm{x}}_{2}^{0} \geq 0$

The optimal solution of (4.3) is

$\underline{x}_{1}^{0}=9.4, \underline{x}_{2}^{0}=1.8$

Similarly the optimal solution of (4.4) is

$\overline{\mathrm{x}}_{1}^{0}=13.1, \overline{\mathrm{x}}_{2}^{0}=2.7$

Solving (4.1) for $\mathrm{r}=1$

$\operatorname{Max} \mathrm{z}_{1}=\left(\underline{\mathrm{x}}_{1}^{1}, \overline{\mathrm{x}}_{1}^{1}\right)+4\left(\underline{\mathrm{x}}_{2}^{1}, \overline{\mathrm{x}}_{2}^{1}\right)$
$\operatorname{Max} \mathrm{z}_{2}=\left(\underline{\mathrm{x}}_{1}^{1}, \overline{\mathrm{x}}_{1}^{1}\right)+5\left(\underline{\mathrm{x}}_{2}^{1}, \overline{\mathrm{x}}_{2}^{1}\right)$

Subject to

$3\left(\underline{\mathrm{x}}_{1}^{1}, \mathrm{x}_{1}^{1}\right)+\left(\underline{\mathrm{x}}_{2}^{1}, \overline{\mathrm{x}}_{2}^{1}\right) \leq(31,41)$

$\left(\underline{\mathrm{x}}_{1}^{1}, \overline{\mathrm{x}}_{1}^{1}\right)+7\left(\underline{\mathrm{x}}_{2}^{1}, \overline{\mathrm{x}}_{2}^{1}\right) \leq(23,31)$

$\underline{x}_{1}^{1}, \bar{x}_{1}^{1}, \underline{x}_{2}^{1}, \bar{x}_{2}^{1} \geq 0$.

There are two auxiliary crisp MOLPP corresponding to $\mathrm{r}=1$

$\operatorname{Max} \mathrm{z}_{1}=\underline{\mathrm{x}}_{1}^{1}+4 \underline{\mathrm{x}}_{2}^{1}$

$\operatorname{Max} \mathrm{z}_{2}=2 \underline{x}_{1}^{1}+5 \underline{x}_{2}^{1}$

Subject to

$3 \underline{x}_{1}^{1}+\underline{x}_{2}^{1} \leq 31$

$\underline{x}_{1}^{1}+7 \underline{x}_{2}^{1} \leq 23$

$\underline{\mathrm{x}}_{1}^{1}, \underline{\mathrm{x}}_{2}^{1} \geq 0$,

and

$\operatorname{Max} \mathrm{z}_{1}=\overline{\mathrm{x}}_{1}^{1}+4 \overline{\mathrm{x}}_{2}^{1}$

Max $z_{2}=2 \bar{x}_{1}^{1}+5 \bar{x}_{2}^{1}$

Subject to

$3 \overline{\mathrm{x}}_{1}^{1}+\overline{\mathrm{x}}_{2}^{1} \leq 41$

$\overline{\mathrm{x}}_{1}^{1}+7 \overline{\mathrm{x}}_{2}^{1} \leq 31$

$\overline{\mathrm{x}}_{1}^{1}, \overline{\mathrm{x}}_{2}^{1} \geq 0$.

The optimal solution of (4.8) is

$\underline{x}_{1}^{1}=9.7, \underline{x}_{2}^{1}=1.9$

Similarly the optimal solution of (4.9) is

$\overline{\mathrm{x}}_{1}^{1}=12.8, \overline{\mathrm{x}}_{2}^{1}=2.6$

Now using optimal solutions (4.5), (4.6), (4.10) and (4.11) in formula:

$\underline{x_{j}}=\left(\underline{x_{j}^{1}}-\underline{x_{j}^{0}}\right) r+\underline{x_{j}^{0}}$

$\overline{x_{j}}=\left(\overline{x_{j}^{1}}-\overline{x_{j}^{0}}\right) r+\overline{x_{j}^{0}}$

to get

$\underline{\mathrm{x}_{1}}=(9.7-9.4) \mathrm{r}+9.4=(0.3 \mathrm{r}+9.4)$

$\overline{\mathrm{x}_{1}}=(12.8-13.1) \mathrm{r}+13.1=(-0.3 \mathrm{r}+13.1)$

$\underline{\mathrm{x}_{2}}=(1.9-1.8) \mathrm{r}+1.8=(0.1 \mathrm{r}+1.8)$

$\overline{\mathrm{x}_{2}}=(2.6-2.7) \mathrm{r}+2.7=(-0.1 \mathrm{r}+2.7)$

Thus optimal solution is

$\tilde{\mathrm{x}}_{1}=\left(\underline{\mathrm{x}_{1}}, \overline{\mathrm{x}_{1}}\right)=(0.3 \mathrm{r}+9.4,-0.3 \mathrm{r}+13.1)$ and

$\tilde{\mathrm{x}}_{2}=\left(\underline{\mathrm{x}_{2}}, \overline{\mathrm{x}_{2}}\right)=(0.1 \mathrm{r}+1.8,-0.1 \mathrm{r}+2.7)$

The optimal values of objective functions are:

$\mathrm{z}_{1}=\left(\underline{\mathrm{x}_{1}}, \overline{\mathrm{x}_{1}}\right)+4\left(\underline{\mathrm{x}_{2}}, \overline{\mathrm{x}_{2}}\right)$

$=\{(0.3 r+9.4,-0.3 r+13.1)$ 


$$
\begin{gathered}
+4(0.1 \mathrm{r}+1.8,-0.1 \mathrm{r}+2.7)\} \\
=(0.7 \mathrm{r}+16.6,23.9-0.7 \mathrm{r})
\end{gathered}
$$

and

$$
\begin{aligned}
\mathrm{z}_{2}= & 2\left(\underline{\mathrm{x}_{1}}, \overline{\mathrm{x}_{1}}\right)+5\left(\underline{\mathrm{x}_{2}}, \overline{\mathrm{x}_{2}}\right) \\
= & \{2(0.3 \mathrm{r}+9.4,-0.3 \mathrm{r}+13.1) \\
& +5(0.1 \mathrm{r}+1.8,-0.1 \mathrm{r}+2.7)\} \\
= & 2(0.3 \mathrm{r}+9.4,-0 .+13.1)+5(0.1 \mathrm{r}+1.8,-0.1 \mathrm{r}+2.7) \\
= & (27.8+1.1 \mathrm{r}, 39.7-1.1 \mathrm{r}) .
\end{aligned}
$$

\section{CONCLUSION}

Senthilkumar and Rajendran [1] have represented the FLPP in parametric form. In this paper, we have represented MOFLPP in parametric form. Ranking function can be used in parametric form. The method is illustrated with the help of numerical example.

\section{ACKNOWLEDGMENT}

The author(s) express their hearty thanks and gratefulness to all those scientists whose masterpieces have been consulted during the preparation of the present research paper.

\section{REFERENCES}

[1] Senthilkumar, P., and Rajendran, G. 2010. On the Solution of Fuzzy Linear Programming Problem, International Journal of Computational Cognition, 8: 4547.

[2] Bellman, R.E., and Zadeh. L.A. 1970. Decision making in a fuzzy environment, Management Science, 17: 141164.

[3] Zimmermann, H. J. 1978. Fuzzy programming and linear programming with several objective functions, Fuzzy Sets and System, 1: 45-55.

[4] Delgado, M., Verdegay, J. L., and Vlla, M. A. 1989. A general model for fuzzy linear programming, Fuzzy Sets and System, 29: 21-29.
[5] Fang, S. C., and Hu, C. F. 1999. linear Programming with fuzzy coefficient in constraint, Comput. Math. Appl., 37: 63-76.

[6] Maleki, H.R., Tata, M., and Mashinchi, M. 2000. Linear programming with fuzzy variable, Fuzzy Sets and System, 109: 21-33.

[7] Allahviranloo, T., Lotfi, F.H., Kiasary, M. Kh., Kiani, N A., and Alizadeh., L. 2008. Solving Fully Fuzzy Linear Programming Problem by the Ranking Function, Applied Mathematical Sciences, 2: $19-32$.

[8] Kumar, A., and Kaur, J. 2011. A New Method for Solving Fuzzy Linear Programs with Trapezoidal Fuzzy Numbers, Journal of fuzzy set valued analysis, 2011: Article ID jfsva-00102, 1-12 doi:10.5899/2011/jfsva00102.

[9] Roseline, S.S., and Amirtharaj, E. C. H. 2012. Different strategies to solve fuzzy linear programming problems, Recent Research in Science and Technology, 4(5): 1014.

[10] Cadenas, J. M., and Verdegay, J. L. 1997. Using fuzzy numbers in linear programming, IEEE, Transactions on Systems, Man and Cybernetics-Part B: Cybernetics 27:1016-1022.

[11] Stanciulescu, C., Fortemps. Ph., Installe. M., and Wertz. V. 2003. Multiobjective fuzzy linear programming problems with fuzzy decision variables, European Journal of Operational Research 149: 654-675.

[12] Cadenas, J. M., and Verdegay, J. L. 2000. Using ranking function in multi objective fuzzy linear programming, Fuzzy set and system, 111: 47-53.

[13] Klir, G. J., Clair, V.S., and Yuan, B. 1997. Fuzzy set theory: foundation and application. Printice-Hall Inc.

[14] Senthilkumar. P., and Rajendran, G. 2009. Solution of fuzzy linear system by using fuzzy centre, Applied Mathematical science, 3: 2411-2419. 